\title{
DECLINAZIONI DEL COMICO NEI RACCONTI DI STEFANO BENNI
}

\author{
MONICA BORIA \\ (Nottingham Trent University)
}

\begin{abstract}
Stefano Benni's work, a blend of biting social satire and fantasy, has won him the reputation of Italy's leading humorous writer. A prolific columnist and author of novels, short stories, poems, and plays, Benni has been playing with language and literary genres since his debut in the late 70s, becoming one of the early Italian writers to engage with postmodern humorous practices. In this article I discuss Benni's collections of short fiction as an instance of these practices and attempt to evaluate their place in the author's wider oevre.
\end{abstract}

Dal successo degli esordi, alla metà degli anni Settanta, con i corsivi satirici su il manifesto ai romanzi best-seller degli anni Ottanta, Stefano Benni (1947-) si è guadagnato il titolo di classico dell'umorismo italiano di oggi. Autore versatile di racconti, romanzi, ballate, pastiche e pezzi teatrali Benni rimane difficile da collocare nel panorama letterario contemporaneo, sia per il suo eclettismo che per gli esiti alterni di scrittura e verve comica.

Lo studio di Tani (1990:129-146), uno dei primi sulla narrativa degli anni Sessanta e Ottanta a menzionare i lavori di Benni, include la sua opera in una sezione dedicata agli "accumulatori" un gruppo di autori (fra cui Tondelli, Busi, Cavazzoni) la cui caratteristica saliente è rappresentata dalla proliferazione picaresca di personaggi e storie surreali. In questi autori l'esuberanza della 
narrazione e l'uso dei dispositivi offerti dal fantastico e dal comico sono impiegati per commentare la società contemporanea: il loro tono è tinto di sarcasmo e i giochi di parole divertenti e provocatori. Di Benni si sottolinea il gioco allusorio con la tradizione letteraria, sia nell'impianto narrativo che rielabora vari generi letterari, sia nella parodia dei classici. Altri critici, fra cui La Porta (1995:183187), Sinibaldi (1997:27-34) e Fofi (Benni e Fofi, 1999), hanno dato più importanza ai contenuti politici del lavoro di Benni, alla sua carica satirica; altri ancora, sopratutto quelli operanti in Nord America come Capozzi (1993:205-222), Ceserani (1994:369-384) e Degli Esposti (1995:99-105), hanno posto l'accento sui suoi tratti postmoderni e cioè l'uso della citazione parodica, l'espressionismo linguistico e la commistione di 'high culture' e 'popular culture'. Anche i pericoli e i difetti della scrittura benniana sono stati variamente individuati: gli eccessi accumulatori che diventano a tratti verbosità, la satira a oltranza che si tinge di moralismo consolatorio, la ricorrenza di meccanismi narrativi e del comico che conduce al manierismo.

È difficile negare che i romanzi di Benni, dopo quello di esordio Terra! (Feltrinelli, 1983), abbiano in vario modo ripresentato temi e forme simili, adottando strutture formulaiche da letteratura giovanilistica e di consumo. Essi mantengono tuttavia una loro originalità sia per le bizzarre trovate narrative e l'efficace contaminazione linguistica e di generi, che per i vari divertissements - racconti nel racconto, pastiche, canzoni ecc. - su cui i romanzi sono costruiti.

La strutturazione narrativa e la comicità sferzante, 'da battuta' tipici di Benni trovano infatti la loro forma ideale nel componimento breve. Le qualità di sintesi e pregnanza spesso richieste ad un racconto, permettono a Benni di tenere a freno 
l'istinto accumulatorio e onnivoro della sua fantasia e di massimizzare l'effetto comico, dei cui meccanismi di attivazione tratterrò fra poco.

Della specifica interazione fra racconto e romanzo, in epoca moderna e contemporanea, si sono occupati in diversi e con conclusioni simili, pur nelle diverse sfaccettature. De Meijer (1984:759-847), ad esempio, con riferimento alle raccolte narrative italiane dal Verismo a Calvino, sottolinea il progressivo allontanamento dalla tradizionale cornice alla Boccaccio in favore del criterio tematico, e vede nel romanzo "l'unico inquadramento organico" delle novelle di oggi, uno degli esiti in cui si incontra "la scelta fra il narrar breve e il narrar lungo" (De Meijer, 1984:791).

Guglielmi (1989:607) sostiene che la fortuna del romanzo a cornice e il crescente ricorso alle forme di novella e racconto nel Novecento, siano da attribuire ai ben noti sintomi di crisi e spaesamento nello scrittore moderno, al quale la conoscenza del reale appare frammentaria: "nelle sue forme epiche - realistiche o naturalistiche - il romanzo tende alla raffigurazione di un mondo, di una totalità di eventi. Novella e racconto, al contrario, considerano per lo più solo un tratto significativo di una storia. La loro illuminazione del mondo è parziale" (Guglielmi, 1989:607).

Con l'arrivo della cosiddetta cultura di massa e del postmodernismo, la costante erosione delle gerarchie di generi e sottogeneri letterari e l'influenza sulla letteratura di generi altri quali il cinema, la musica rock o i fumetti sono sono dati pressochè 'accettati' dagli anni ottanta. ${ }^{1}$ Questa evoluzione si riflette anche nel

1 Il non-romanzo di Calvino Se una notte d'inverno un viaggiatore (1979) e soprattutto il bestseller di Eco, Il nome della rosa del 1980 sono considerati quasi dei manifesti del postmoderno letterario in Italia, contribuendo all'avvio di un discusso rinnovamento ancora in corso. Sul dibattito del postmoderno in Italia si vedano in particolare: Remo Ceserani, Raccontare il postmoderno, Torino: Bollati Boringhieri, 1997, Pischedda, 'Modernità del postmoderno', in 
campo del racconto: i filoni del fantastico ( fantasy, fantascienza ecc.) e del giallo hanno ricevuto un certo impeto (Guagnini, 1993:115-131; Schiavo, 2002) e i recenti lavori dei giovani autori ('cannibali' e non) hanno incarnato forse più chiaramente di altri questo cambiamento.

Nell'ambito specifico dell'interazione fra il comico e la narrativa (specie breve), c'è chi ha parlato di "un'invadenza generalizzata del comico" e della "fine della separazione netta fra comico e tragico e il drastico rimescolamento dei loro rispettivi confini" (Turchetta, 2004:106). Le conseguenze di questa tendenza sono state duplici: da un lato si è assistito all'introduzione di meccanismi e temi tipici del registro comico e 'basso' nella letteratura cosiddetta 'seria', dall'altro si è registrata una sottile ma inesorabile erosione dello statuto del comico stesso, che risulta relativizzato, se non addirittura neutralizzato.

$\grave{E}$ in questo contesto che i lavori di Benni vanno inseriti per comprenderne sia l'originalità che il successo. Va riconosciuta a Benni l'audacia di esser stato fra $\mathrm{i}$ primi in Italia a giocare allo scoperto con delle categorie, quella del comico e quella della cultura di massa, per affrontare un discorso politico e letterario serio. In questo senso alcuni suoi corsivi apparsi su il manifesto alla fine degli anni Settanta e i pezzi 'a la maniere dé' rimangono a mio avviso fra le cose più riuscite della sua vena parodico-satirica ${ }^{2}$ e

Belfagor, 5 (1997):579-588, Margherita Ganeri, Postmodernismo, Milano: Editrice Bibliografica, 1998 e Monica Jansen, Il dibattito sul postmoderno in Italia. In bilico tra dialettica e ambiguità, Firenze: Cesati, 2002.

2 Particolarmente efficaci i pastiche e le parodie di poeti come Montale, Pascoli, Ginsberg ecc. contenuti nelle raccolte Prima o poi l'amore arriva, 1981 e Ballate, 1991. Ne ho discusso nel mio articolo 'Echoes of Counterculture in Stefano Benni's Humour', Romance Studies Journal, 23 (March 2005): 29-42. 
andrebbero inseriti a pieno titolo in una storia del corsivo moderno, ancora tutta da scrivere. ${ }^{3}$ I racconti qui proposti costituiscono un esempio dell'intento parodico-satirico riscontrabile nell'opera di Benni e un campione rappresentativo della capacità di Benni di modulare il comico e di orchestrare lingua e architettura narrativa. Vogliono allo stesso tempo essere un esempio di quella tendenza della produzione letteraria contemporanea alla commistione di generi e di cultura 'alta' e 'bassa' e alla metaletterarietà tipica dell'episteme postmoderna.

Nella sua prima raccolta, Bar Sport (1976), il bozzetto umoristico che introduce il primo personaggio ('Il tecnico') è costruito sulle cadenze linguistiche e narrative del documentario televisivo, che fanno dell'intera raccolta un moderno bestiario. Gli stilemi della favola sono invece rielaborati in modo originale in alcuni racconti delle raccolte successive, come nel pezzo 'Underground' (Bar Sport Duemila, 1997) e 'La traversata dei vecchietti' (Il bar sotto il mare,1987). La contaminazione con i generi non-letterari, soprattutto cinema e fumetti, ritorna con forza nella collezione del 1987 e sottende all'organizzazione stessa della raccolta, in sé una costruzione complessa di allusioni. Il racconto con cui concluderò questa ricognizione, 'Papà va in TV' (L'Ultima lacrima, 1994), è un agghiacciante esempio di umorismo nero, emblematico del tono caratterizzante la raccolta, organizzata senza altro principio che appunto quello del sarcasmo.

"Un tempo c'era il caffè letterario. Poi arrivò Stefano Benni. E da allora c'è il bar sport." Così si apre l'articolo di Magistà su La Repubblica in occasione della imminente uscita di Bar Sport

3

Si veda al proposito Robin Pickering-Iazzi, 'Pirandello and Buzzati: A Profile of the Short Story Elzeviro', Quaderni di Italianistica, 8 (1987): 194-215. 
Duemila (Feltrinelli, 1997) nonchè della ristampa di Bar Sport (Mondadori, 1976) presso Feltrinelli. Al tono scherzosamente celebratorio di questo (come di altri) incipit giornalistici, seguono di solito precisazioni più sobrie: "l'abisso che separa la raffinata conversazione parigina dal surreale chiacchiericcio della provincia emiliana è stato superato da Benni col salto mortale dell'umorismo", l'arma che gli permette di ritornare con divertito distacco, venti anni dopo, su quei "bozzetti umoristici, a volte perfino un po' deboli”' (Magistà, 1997:67).

Se nelle successive raccolte di racconti Benni affina tecniche linguistiche e impianto narrativo, presentando una vasta gamma sia di 'generi' di racconto che di tonalità del comico, cominciare questa ricognizione proprio dalla sua opera prima, Bar Sport, mi sembra appropriato per due motivi. Da un lato - in un'ottica interpretativa interna - questa raccolta funziona da matrice per quelle successive, e più sofisticate, del 1987 e del 1997, come è tipico del modus operandi benniano. ${ }^{4}$ Dall'altro lato - considerando l'opera di Benni stessa come prodotto della cultura contemporanea - Bar Sport e il successivo Bar Sport Duemila, forniscono una testimonianza, a tratti irresistibile, dell'evoluzione dell'identità italiana. ${ }^{5}$

4 Come è stato notato da più parti, Benni ha la tendenza a rielaborare forme, contenuti e fonti varie in un gioco intertestuale continuo che include anche i suoi stessi testi. Cf. Corrado Augias, 'Sinistra dalle mani vuote', Il Venerdì di Repubblica, 9 febbraio 1996; Carol O'Sullivan, 'Intercomically Textual: Stefano Benni's «esercizi di stile»', Contributo presentato alla conferenza 'Senses of Humour', Cambridge, 18 March 2000.

È opinione diffusa che la letteratura di massa, più che quella 'alta', rappresenti una importante fonte per determinare usi, costumi, idée ecc. di una certa società in un dato periodo storico. Cf. Tony Bennett, 'Marxism and Popular Fiction', in Popular Fictions. Essays in Literature and History, P. Humm, P. Stignant, P. Widdowson (eds), Londra/New York: Methuen, 1986: 237265. 
Bar Sport $^{6}$ può essere definito una raccolta di racconti a cornice: il bar, come punto di incontro e di passaggio, fornisce l'ambientazione ideale per rappresentare i più svariati tipi umani e Benni sceglie tutta una serie di personaggi - 'Il professore', 'Il tecnico', 'Pasquale il barbiere', 'Il vero pescatore', 'Il playboy da bar' ecc. - che costituiscono esempi tipici di varia umanità di provincia. Il bar come luogo metaforico di socializzazione $\mathrm{e}$ affabulazione sottende invece alla cifra stilistica di molte storie, che presentano il ritmo e l'andamento narrativo tipico del racconto orale.

I ventisei pezzi che costituiscono la raccolta sono preceduti da una 'Introduzione' umoristica, sotto forma di excursus storicoantropologico, sull'origine del bar:

L'uomo primitivo non conosceva il bar. Quando la mattina si alzava, nella sua caverna, egli avvertiva subito un forte desiderio di caffè. Ma il caffè non era ancora stato inventato e l'uomo primitivo aggrottava la fronte, assumendo la caratteristica espressione scimmiesca. Non c'erano neanche bar. Gli scapoli, la sera, si trovavano in qualche grotta, si mettevano in semicerchio e si scambiavano botte di clava in testa secondo un preciso rituale. [...] Ma questo primo tentativo di bar fu un fallimento. Non esistevano la moviola, il vistoso sgambetto, il secco rasoterra [...] $(B S, 7)$

6 Bar Sport, Milano: Mondadori, 1976 (1995). D'ora in avanti BS. 
Questa pseudo-introduzione, utilizzando ironicamente gli stilemi della narrazione scientifica, stabilisce fin da subito il tono dell'intera raccolta e l'intento di produrre un moderno bestiario.

Solo in alcuni pezzi Benni riesce a trovare un equilibrio efficace fra $\mathrm{i}$ vari livelli messi in gioco e a mantere alta la coerenza fra la cornice del bar, la verve affabulatoria tipica dell'oralità e il tono da ironico bestiario. Uno di questi racconti è 'Il tecnico', il bozzetto che ritrae l'irritante esperto di tutto. Questa figura tipica della 'fauna' del bar è descritta utilizzando cadenze linguistiche e narrative tipiche del documentario televisivo o della prosa scientifica divulgativa. Ecco l'inizio:

Il tecnico da bar, più comunemente chiamato "tennico" o anche "professore", è l'asse portante di ogni discussione da bar. Ne è l'anima, il sangue, l'ossigeno. Si presenta al bar dieci minuti prima dell'orario di apertura: è lui che aiuta il barista ad alzare la saracinesca. Il suo posto è in fondo al bancone, appoggiato con un gomito. Lo riconoscerete perché non si siede mai e porta l'impermeabile e cappello anche d'estate. Dal suo angolo il tecnico osserva e aspetta che due persone del bar vengano a contatto. Non appena una delle due apre bocca, lui accende una sigaretta e piomba come un rapace sulla discussione. Nell'avvicinarsi, emette il verso del tecnico: "Guardi, sa cosa le dico", e scuote la testa. [BS, p.. 33]

Espressioni che riecheggiano lo stile dei documentari TV, come "più comunemente chiamato", sono utilizzate in modo coerente in tutto il racconto per tracciare il ritratto del personaggio: alla 
descrizione sopra riportata seguono infatti precisazioni del tipo "normalmente si ciba solo di aperitivi", "vive in simbiosi con un altro personaggio" ed altre simili (34-35). Il tono pseudodivulgativo, reso immediatamente evidente da queste espressioni, è insomma un espediente stilistico funzionale alla caratterizzazione del personaggio del tecnico, che viene gradualmente ad identificarsi con un qualche esemplare degno della nostra attenzione.

Il lettore scopre infatti le sue abitudini ("si presenta"), il suo habitat preferito ("il suo posto"), il suo aspetto ("lo riconoscerete"), infine i suoi rituali ("osserva e aspetta", "accende una sigaretta"). Il paragone con il rapace e l'espressione "emette il verso", completano l'identificazione, assimilando l'esordio verbale del tecnico a un gracchio di vuota saccenza. Che l'esperto, in realtà, non dica che sciocchezze apparirà ben presto in modo chiaro, infatti "cita largamente dal latino: sine qua non (siamo quà noi) o fiat lux (faccia lei). Usa verbi col congiuntivo tattico: se me lo dicevaste prima, anderei" (35).

I battibecchi calcistici fra il tecnico e il suo incompetente rivale, l'elusivo "uomo col cappello", sfociano nella zuffa conclusiva, animalesca lotta per la supremazia del territorio del bar: "[...] e in breve tempo si azzuffano. La gente li separa. Il tecnico si allontana con aria di superiorità. L'uomo col cappello, rimasto padrone del campo, dichiara che l'Italia non vincerà mai uno scudetto finché continua a tenere Pelè in porta. Viene preso, pestato, e mandato via col camion del rusco" $(B S, 38)$.

La conclusione presenta un'altra caratteristica del bestiario: la morale. L'uomo col cappello ha osato sfidare l'esperto ma la sua grossolana incompetenza non convince e viene dunque allontanato dai frequentatori del bar. Se consideriamo tuttavia il fatto che il tecnico stesso non è in realtà che un altezzoso contafrottole col 
dono dell'eloquenza, la morale della storia assomiglia più ad un'antimorale: è l'abilità nel raccontare storie che suscita ammirazione fra gli astanti e il ghigno divertito del narratore.

Questa idea sembra confermata dallo stesso Benni in un'intervista de La Repubblica (Malatesta, 1992:27) in cui ricorda le notti brave bolognesi negli anni Settanta:

Si stava in giro fino alle sei di mattina. Vitellonismo e interclassismo, gli operai accanto ai figli dei borghesi. Il legante erano la cordialità e l'umorismo, i bar come centro di produzione, come serbatoio del comico autentico, spontaneo. Decine di raccontatori che facevano dei grandi meló, con trame intrecciate tra loro, a puntate. Un'invenzione verbale continua, storie di caccia, di pesca, di sesso: tenevano inchiodato l'uditorio per due, tre ore di fila. Se vai nei bar ora, ti ripetono l'ultima battuta sentita in televisione, magari è divertente, ma è di riporto.

Se Bar Sport è anche un omaggio, seppure ironico, a un mondo che Benni ha conosciuto e apprezzato, si sarebbe tentati di spiegare il tono sarcastico che domina Bar Sport Duemila in termini di amara nostalgia. L'evoluzione dell'Italia da paese pre-moderno a postmoderno è agli occhi di Benni una gran degenerazione, rappresentata simbolicamente nella smania di novità e di ostentatazione descritta nel racconto che apre la raccolta, intitolato 'Psicopatologia del bancone da bar':'

7 Bar Sport 2000, Milano: Feltrinelli, 1997: 7. D'ora in poi BS2. 
[...] dei bellissimi banconi di legno perfettamente funzionanti vengono sostituiti con banconi a "esse", a labirinto, pralinati con lapislazzuli, in materiali che vanno dalla bachelite arancione al vetro blindato. Gli stili passano dal rococò-maya al neotorronico bugnato, dal liberty-linoleum al Barbie-Goodzilla, dal CheopeChippendale al post-Benito, dal gotico-zotico al Luigi$\mathrm{X}$-Files, dall'assiro-bullonese al techno-etrusco. (BS2:

7)

Come il titolo lascia intuire, questi banconi anni Novanta rappresentano malsane categorie dello spirito nazionale: dal neokitsch ("pralinati con lapislazzuli", "neotorronico") al machismo politico ("post-Benito", "assiro-bullonese") passando per l'incanto del fascino straniero ("liberty-linoleum", "Luigi-X-files"). Ma la degenerazione è evidente soprattutto nei nuovi avventori, personaggi sinistri e neurotici, come ad esempio 'Il Diditì, o il drogato da telefonino', nel quale Benni ironizza sull'uso (o abuso) del telefono cellulare fra gli italiani. L'apice di neurosi e falsità si raggiunge ne 'Il Bar Fico', dove troviamo l'inedito personaggio di "Gastone l'animatore". Questo personaggio è funzionale alla derisione delle pretenziose notti brave di gruppi con ambizioni, frustrate, di evadere dalla vita di provincia. Infatti dopo una serie di stravaganti quanto improbabili itinerari, Gastone suggerisce un prosaico "andiamo tutti a casa della Titti a vedere Sanremo e mia mamma ci fa le sfrappole" (BS2: 27). D'altro canto la mera presenza di un animatore ufficiale tradisce la mancanza di quella spontaneità a cui Benni si riferiva sopra, un'assenza che sembra scaturire da una generale perdita di genuinità nei rapporti interpersonali, descritti da Benni con sarcasmo: 
Mentre si saluta e si bacia il primo conoscente, già con la mano si fa cenno al secondo e si strizza l'occhio al terzo. Per uno strano contrappunto, queste espressioni d'affetto e cameratismo vengono per lo più accompagnate da spiritosi epiteti quali "brutto bastardo!", "eccoti quà, vecchia checca!" oppure "stronza, dov'eri finita?" [...] Il vero habitué del Bar Fico poi, non solo saluta fragorosamente, ma piange di commozione, stritola mani, bacia sensualmente, dopodiché si apparta in un angolo con un conoscente esternandogli l'odio per tutti i presenti, l'insofferenza per queste recite smancerose, e la noia di doversi recare lì tutte le sere, mentre nei bar di Manhattan o di Marbella c'è tutta un'altra atmosfera. (BS2: 23-24)

Bar Sport 2000 risulta tuttavia una raccolta per molti versi più sofisticata della prima, con trovate narrative più fantasiose e una varietà di registri linguistici e tonalità del comico più elaborate; il pericolo di una tale operazione è comunque evidente. Benni finisce in più occasioni per rifare il verso a se stesso, oltre che alle manie e al provincialismo italiani. Ne è un esempio il breve excursus sulla pasticceria, ispirato al famoso racconto 'La Luisona', la rassicurante pasta di Bar Sport che "si trovava nella bacheca dal 1959." La Luisona infligge dolori inauditi all'ignaro avventore di passaggio che la mangia. La successiva "Palugona" del bar "peso" e le "brioche invisibili a occhio nudo" (BS2: 20-22) del bar sofisticato sono una variazione manieristica sul tema.

Il personaggio del tecnico sopra descritto è un altro esempio di questa caduta di tono. In Bar Sport Duemila l'interesse di Benni 
non è più focalizzato su un certo tipo umano e sulle dinamiche sociali scatenate dal personaggio - interesse che rendeva appropriato e divertente l'impiego degli stilemi pseudo-scientifici di cui ho detto. Con 'Il neo tecnico da bar' Benni intende colpire da una parte la Tv spazzatura e dall'altra la volubilità e il vuoto di ideali dell'Italia contemporanea. Il tecnico di oggi è definito "computerizzato e satellitare" e Benni si dilunga nel descrivere la sua competenza tecnologico-calcistica giocando con neologismi piuttosto scontati. ${ }^{8}$ Anche in questo racconto si narra lo scontro fra $\mathrm{i}$ due esperti - scontro che assume le forme di conferenza pubblica con tanto di ospite d'onore e rinfresco finale, ma il racconto si conclude in modo molto più amaro. Il famoso calciatore, oggetto della disputa, è dimenticato non appena dalla TV giunge la notizia di un nuovo possibile acquisto, l'islandese Odin:

Allora, faccio questi palleggi o no? - chiese il nuovo Pelè. Ma nessuno lo ascoltava più. La discussione verteva ormai sulla cifra, cioè se Odin valeva veramente dieci miliardi, e venivano fatti confronti col costo di Baggio, con duecento ettari a frutteto e con il deficit dell'Inps. Già era nata una nuova polemica, un nuovo dibattito, un nuovo dilemma filosofico, nell'ultima vera passione civile che riscalda il nostro popolo. (BS2: 99)

Il procedimento metonimico adottato per alludere alla chiacchiera da bar - che spazia dal calcio, all'agricoltura e all'economia appare più raffinato e di più sicuro effetto comico che nel

8 L'era del "paleotecnico" è definita ad esempio "Catodico Primario". BS2: 92-93. 
precedente lavoro; ${ }^{9}$ l'amaro moralismo della chiusura si distacca invece nettamente dall' anti-morale tra le righe del pezzo del ' 76.

Il crescente pessimismo che traspira dai lavori di Benni dagli anni Ottanta in poi è legato alla nota parabola di disillusione politica della sua generazione, che dall'esperienza deludente del Movimento del '77 e dallo shock del terrorismo, fino alla crisi del cosiddetto 'riflusso' degli anni ottanta, si è confusamente interrogata sulla questione dell'impegno nel decennio successivo. La posizione di Benni come intellettuale di sinistra è passata per le fasi della militanza, della critica e auocritica, e infine del distacco. Dagli anni Novanta il suo impegno si è concretizzato in iniziative $\mathrm{e}$ atteggiamenti che raramente hanno coinciso con quelli della sinistra ufficiale. Attraverso collaborazioni con personaggi del teatro spesso controversi come Dario Fo e Beppe Grillo, iniziative culturali con scuole, associazioni no-profit e gruppi impegnati nel sociale, ${ }^{10}$ Benni ha dato forma nuova al suo lavoro 'politico'. Pur continuando le collaborazioni a testate di sinistra come il manifesto e La Repubblica, la sua attività di scrittore impegnato si è arricchita della collaborazione con i giovani artisti del Teatro dell'Archivolto (i Broncoviz televisivi) e il regista Giorgio Gallione, con attori come Lucia Poli, Angela Finocchiaro e Paolo Rossi. È interessante notare che l'eclettismo del suo impegno - che include fra gli altri anche un film e concerti di poesia e musica jazz - ha escluso

9 In $B S$ quest'idea era resa con un'enumerazione piuttosto semplice: "Di cosa parla un tecnico? Di calcio, di sport in genere, di politica, di morale, di macchine, di agricoltura, di prezzi della frutta, di diabete, di sesso, di trattori, di cinema, di imbottigliamento, di spionaggio. In una parola di tutto." (34).

10 Per informazioni più dettagliate su alcune di queste attività si veda: http://www.stefanobenni.it/fabula/. 
sistematicamente la televisione, una delle arene nelle quali Benni si è sempre rifiutato di apparire e che ha costantemente attaccato fin dagli anni de il manifesto. Di questo distacco programmatico che si riflette in brani dai toni feroci, parlerò nell'ultima parte di questo contributo.

A conclusione di questo primo excursus sulle raccolte del Bar Sport, vorrei soffermarmi su un racconto di Bar Sport Duemila, che rappresenta a mio avviso uno dei migliori delle raccolta e le cui forme appaiono alquanto diverse rispetto a tentativi simili del precedente Bar Sport. Nel racconto 'Underground'11 Benni compie un rovesciamento di prospettiva e ci descrive un momento della normale routine di un qualsiasi bar della stazione dal punto di vista degli scarafaggi, protagonisti del racconto. La storia dei "Guerrieri Neri" è una favola surreale in cui si ripropongono, con sottili effetti comici, alcuni dei topoi classici del romanzo cavalleresco e della fiaba. Ne risulta una favola metropolitana dalla parte dei più deboli, ovvero gli insetti. Gli scarafaggi intraprendono un pericoloso viaggio per liberare la principessa Bea, rimasta prigioniera nella teca delle paste sul bancone del bar. Con una mosca come guida, il gruppo raggiunge l'affollato bar perdendo tuttavia un compagno durante l'attraversamento della strada, il trafficato "deserto grigio". La conclusione ha un doppio lieto fine: la liberazione di Bea e la vendetta sugli umani.

11 BS2: 108-118. Va notato che l'intento di produrre un moderno bestiario permane anche nella seconda raccolta e che tra le due raccolte del Bar Sport un vero e proprio bestiario è rappresentato da Stranalandia (Feltrinelli, 1984), un libro 'per ragazzi' dove le belle illustrazioni di Pirro Cuniberti danno corpo alla fantasiosa fauna benniana, creando uno degli esempi migliori di quel processo di ibridazione di realtà e gioco di parole tipico di Benni. È il caso del "Prontosauro" o "Megasaurus interurbanus, animale straordinario, che discende direttamente dai colossali rettili dell'antichità e dalle colossali bollette Sip del nostro tempo." (ediz. 1994: 15), del "Pappagatto" ovvero "Cocomicius technicoloris" (65) e altri. 
In questo, come in altri dei suoi migliori racconti, l'estro narrativo di Benni risiede nell'abile equilibrismo fra i vari livelli evocati: lo stile favolistico, il tono umoristico e la critica sociale. L'ambiguità che caratterizza l'ambientazione e l'identità dei protagonisti ad apertura di racconto è costruita attentamente con l'intenzione di ricreare attorno a questi personaggi - insetti considerati ripugnanti - un'aura mitica:

In quell'angolo tra due pareti la luce giungeva fioca e rossastra, accendendo di bagliori infernali la corazza di Capitan Carabus. La sua ombra si disegnava come quella di un drago sul muro bianco. L'aria calda del tombino faceva vibrare la lunga lancia.

- Ho bisogno di tre volontari per liberare la principessa Bea - disse con voce marziale, che lasciava però trasparire una forte tensione. Dalle file dei Guerrieri Neri si levò un mormorio. Poi il più imponente, il leggendario Scaraffa, orbo e zoppo per le mille battaglie, chiese a nome di tutti:

- E dov'è prigioniera la principessa?

- In un Centopiedi - disse Capitan Carabus.

I Guerrieri Neri scricchiolarono di paura. Entrare in un posto come quello significava morte certa. Molti se la svignarono. Ne restarono solo quattro.

- Come faremo a trovarla? - chiese il giovane Cockroach, che aveva tatuato sul dorso lucido un teschio con la scritta "No future".

- Una Ronzante l'ha vista e ci guiderà sul posto. La principessa è chiusa in una gabbia di vetro, in cima a una Montagna argentata. (BS2: 108) 
L'atmosfera evocata è una contaminazione fra le storie classiche popolate da principesse e cavalieri (o pirati e belle fanciulle), prove da superare in luoghi incantati e pericolosi e le loro rivisitazioni fatte dai fumetti e dai cartoni animati. Questa contaminazione contribuisce ad attivare gradualmente l'effetto umoristico, poi attuato anche attraverso scelte lessicali ed espressioni che 'stonano' col tono epico della narrazione, come "scricchiolarono di paura" e "se la svignarono", o che lasciano intuire la vera dimensione del racconto - i nomi dei personaggi sono in fondo abbastanza eloquenti.

Dopo il disorientamento iniziale, il lettore entra dunque facilmente nel gioco di rovesciamento di prospettiva della storia, dove Benni gioca, sempre più divertito, a carte scoperte. Sentiamo infatti delle sventure familiari della povera mosca, Ronzante, che si rifiuta di entrare nel bar, dicendo di avervi perso la madre e due sorelle "morte invischiate nella Palude Gialla" $(B S 2,109)$. La vita in città traspare sempre più come un mondo terribile e pericoloso per gli animali, un mondo dove gli umani, "i Biscarpe", appaiono spietatamente noncuranti.

Una simile strategia straniante è adottata da Benni nel racconto 'Carmela', parte dell'ultima raccolta La Grammatica di Dio (2007), in cui la protagonista disquisisce con zio Giovanni sulla richiesta di aiuto a Sandrino, un bambino malato. Il lettore scoprirà alla conclusione della storia che Carmela è una gallina e Giovanni il cane di famiglia e il suo aiuto consiste nel sacrificio alla mensa degli umani, al quale la gallina si sottomette con rassegnazione e devozione.

L'ecologismo di Benni e la presa di posizione a favore dei più deboli e degli sconfitti dalla Storia è ricorrente nei suoi lavori: i 
protagonisti di quasi tutti i suoi romanzi sono dei ragazzini, le cui avventure si dipanano in diretto antagonismo con gli adulti o sullo sfondo di un mondo cinico e corrotto che schiaccia i più deboli. In Comici spaventati guerrieri, (1986) un gruppo di ragazzini di periferia indaga sulla morte sospetta di un loro compagno con l'aiuto di un vecchio professore in pensione, ne La Compagnia dei Celestini (1992) dei giovani orfani cercano di sfuggire rocambolescamente al magnate televisivo che vuole riprendere il loro segreto campionato di "pallastrada". In Terra! (1983), invece, la filosofia orientale e la saggezza degli Inca è contrapposta alla presunta superiorità occidentale e fra i protagonisti troviamo un'ape intelligente, al servizio degli umani, che si sacrifica per la riuscita della loro missione spaziale, e un'intero equipaggio di topi altrettanto intelligenti che, ribellatosi alla tirannia degli uomini, soccombe senza speranza. Gli eroi di Benni, siano essi bambini orfani, giovani metropolitani, maghi, insetti o creature di fantasia sono immancabilmente in viaggio alla ricerca di qualcosa $o$ qualcuno e l'impresa assume sempre i connotati di una lotta del bene contro il male. Non si tratta però di una solitaria avventura donchisciottesca: questi eroi, raramente senza macchia, formano di solito un gruppo la cui marginalità e le cui dinamiche 'protestatarie' ma ironicamente disincantate ricordano molto quelle degli 'indiani metropolitani' del Movimento del '77.'

12 Per informazioni più approfondite sul Movimento si vedano: Robert Lumley, States of Emergency. Cultures of Revolt in Italy from 1968 to 1978, Londra: Verso, 1990 e Claudia Salaris, Il movimento del 77. Linguaggi e scritture dell'ala creativa, Bertiolo: AAA Edizioni, 1997. Per una discussione dei romanzi di Benni rimando al mio saggio incluso in: Franca Pellegrini e Elisabetta Tarantino (a cura di), Il romanzo contemporaneo. Voci italiane, Leicester: Troubador, 2005: 43-58. 
In 'Underground' è attraverso il personaggio di Cockroach che Benni allude a quegli anni, alle rivendicazioni sul tempo libero e al fenomeno della larga diffusione delle droghe fra $i$ giovani. Cockroach rivendica il diritto a divertirsi e a godere dell'effimera ma intensa esperienza dello 'sballo', rappresentata da un irresistibile "Tunnel dell'Amore alla crema". Al compagno che si crogiola nel pasticcino ribadendo il suo slogan "no future", Capitan Carabus fa eco con uno sconsolato: "Ormai è perduto. I bignè danno subito assuefazione. Ne ho visti tanti finire così" $(B S 2,117)$.

Le lunghe tirate filosofiche dei topi in agonia o il verboso discorso d'addio dell'ape Sara di Terra! sono a mio avviso felicemente superati da Benni con questa ironia un po' surreale e di battute ad effetto, che ritornano beffardamente nella conlusione di 'Underground', dove al ricongiungimento della principessa Bea al suo cavaliere Carabus, segue il ritorno dell'amico Scaraffa, creduto affogato in una tazzina di caffè:

- Chi sei creatura infernale? - urlò Capitan Carabus, uscendo dalla bottiglia lancia in resta. Ma la voce gli si strozzò in gola.

Davanti a lui c'era Scaraffa!

Il vetro concavo della bottiglia ne aveva ingigantito e deformato l'immagine, ma era proprio il vecchio guerriero. Ingoiato, glassato di succhi gastrici e vomitato, ma vivo!

- Amico mio - disse Carabus abbracciandolo. - È incredibile!

- Beh, - rise Scaraffa - anche nella vita di un piccolo scarafaggio può esserci una grande soddisfazione. (BS2:118) 
Lo stile e gli archetipi della fiaba ${ }^{13}$ rielaborati in questo racconto trovano dei precedenti, anche se in versione semplificata, in altri componimenti della precedente raccolta Il bar sotto il mare (1987), come ad esempio nella storia 'La traversata dei vecchietti' o nella brevissima 'Shimizè'. La prima racconta iperbolicamente della 'impresa' di due nonni di città che vogliono attraversare la strada per raggiungere un parco. Dopo varie 'prove' (strisce pedonali, fingersi morti, dirottamento di bicicletta) e l'arrivo di un 'aiutante'(un terzo vecchietto, in realtà cooptato), riescono ad attraversare tendendo un 'tranello' ad un poliziotto:

- Ah, gli anziani d'oggi! Imprudenti. C'è troppo traffico e siete vecchi e malandati. [...] Da dove vi siete mossi? - disse il poliziotto.

- Da lì - disse Alberto indicando il marciapiede che volevano raggiungere.

- Allora vi ci riporto, e guai se cercate ancora di attraversare - disse il poliziotto.

Così con la scorta della polizia i tre vecchietti riuscirono a passare dall'altra parte e poi arrivarono al giardino.

C'era veramente un bel laghetto. Si trovarono così bene che non riattraversarono mai più. ${ }^{14}$

13 In questa storia sono facilmente riconoscibili le più importanti funzioni della fiaba descritte da Propp (1929): mancanza (principessa); divieto e infrazione (interdizione dal bar); partenza e trasferimento nello spazio; lotta e vittoria; ritorno; persecuzione (di uno degli eroi, Scaraffa); arrivo in incognito e identificazione (Scaraffa); punizione (inflitta agli umani) e ricongiungimento (Carabus e Bea).

Il bar sotto il mare, Milano: Feltrinelli, 1987 (1994): 119. D'ora in poi BMare. 
Anche in questa storia Benni utilizza le forme tradizionali per rovesciarle: se in 'Underground' era la prospettiva ad essere rovesciata, qui sono gli eroi ad essere i villain della situazione. La decostruzione della tradizione letteraria è in parte funzionale alla critica sociale che Benni vuole esprimere e in parte una battaglia a sé. Fin dai tempi della scuola, in cui si divertiva a riscrivere in chiave comica la conclusione di storie e romanzi famosi, l'atteggiamento beffardo di Benni verso la tradizione letteraria italiana, e non, è riconducibile alla sua battaglia nei confronti dello snobismo della cultura 'alta' per la cosiddetta produzione 'bassa'. Questo atteggiamento è facilmente riconducibile allo spirito studentesco della protesta del '68 prima e del ' 77 poi nonché alla più vasta tendenza post-moderna alla citazione e riscrittura parodica. Quest'ultima però non è mai fine a se stessa e le forme canoniche sono straniate o a fini di satira sociale e politica, come nei due racconti sopra descritti o a fini metaletterari, come in 'Shimizè', dove gli archetipi della favola e riminescenze letterarie (il Calandrino di Boccaccio) servono ad un gioco metaletterario e metalinguistico dagli effetti stranianti:

C'era un oshammi shammi che viveva in una weseshammi in cima a una wooba. Venne una notte un oogoro e disse all'oshammi shammi:

-Shimì non voglio né la tua corona né il tuo bastone, voglio la tua shammizé.

- De shimite deé - rise l'oshammi shammi - cerca pure. Se vedi qua nella weseshe la mia shammizé, prendila pure. 
L'oogoro frugò in lungo $\mathrm{e}$ in largo tutta la wesesheshammi e alla fine vide la woolanda e trionfante gridò:

- Shimì, eccola qui, l'ho trovata. [...] (Bmare: 119)

In questa breve storia è la labilità dello statuto referenziale della lingua ad essere preso come nucleo tematico. L'entusiasmo dell'oogoro sarà ben presto deluso quando un terzo personaggio beffardamente smaschererà l'inganno: "Alla luce della luna l'oogoro guardò bene, vide il suo errore e se ne andò tzuke shimite no shimé, triste come chi ha perso il nome delle cose" (Bmare: 120).

Come ricorda Turchetta (2004:112-113), fra i generi e sottogeneri comicizzati la favola e la fiaba sono fra i più sperimentati e hanno attirato autori fra i più diversi, da Manganelli a Malerba e Brancati, producendo una vasta gamma di racconti (fiaba umoristica, erotica, parodia e metafiaba). Come giustamente ha osservato May (1995:83-84) l'impatto del postmodernismo sul racconto di oggi ha portato ad una crescente autoreferenzialità della letteratura, che spesso si caratterizza come tematizzazione dei meccanismi letterari, del 'fare' letteratura: "[the contemporary] short story has a tendency to loosen its illusion of reality in order to explore the reality of its illusion. Rather than presenting itself "as if" it were real - a mimetic mirroring of external reality postmodernist short fiction often makes its own conventions and devices the subject of the story as well as its theme."

La raccolta Il bar sotto il mare rappresenta in questo senso il punto massimo di questa tendenza all'interno dell'opera di Benni. La raccolta è infatti una complessa rete di rimandi letterari (e non), organizzata nella forma classica della cornice boccaccesca, dove il 
novellare è affidato agli avventori del bar. Questi ultimi sono raffigurati in copertina e poi identificati in modo generico in una silhouette con didascalia, posta prima del prologo: troviamo dunque 'il barista', 'il venditore di tappeti', 'primo uomo con cappello', ecc. Alcuni di loro sono chiare icone della cultura di massa (Marilyn Monroe, Elvis Presley, John Belushi) parte di un pantheon personale dell'autore che include anche gli scrittori Edgar A. Poe e Agatha Christie. In primo piano, al centro del ritratto, 'l'uomo con la gardenia' (Sigmund Freud) che invita l'io narrante del prologo a seguirlo nel bar subacqueo, è un chiaro riferimento al viaggio nel subconsio culturale e letterario benniano che il lettore si accinge a compiere.

Come è stato notato da Tani (1990:221-222), molti di questi racconti sono parodie di racconti o opere letterarie ben note, da Melville a Easton Ellis. Questo gioco di rimandi è inoltre rafforzato, in ciascun racconto, dalla presenza di un'epigrafe che illustra sinteticamente il racconto che segue; il succitato racconto 'Shimizè', narrato dalla sirena, è introdotto da una citazione di Karl Kraus che recita: "In nessuna lingua è difficile intendersi come nella propria lingua". Naturalmente abbondano allusioni ironiche alla cultura di massa, soprattutto anglo-americana, spesso rivisitata in chiave nostrana, come nel caso de 'La storia di Pronto Soccorso e Beauty Case', una parodia iperbolica delle tipiche vicende di 'duri' di tanta cinematografia americana.

Un altro critico, Helmut Jacobs (1997:21-37), ha messo l'accento sul fatto che i rimandi intertestuali sono estesi anche all'ambito dell'arte figurativa: la 'foto di gruppo' della copertina ricorda vari dipinti del Novecento, raffiguranti scene di bar, come ad esempio Au rendez-vous des amis (1922) di Max Ernst, una 
ironica rappresentazione di famosi personaggi del Dadaismo e del futuro Surrealismo.

Il racconto con cui concludo questa ricognizione è un pezzo di umorismo nero, piuttosto diverso da quelli discussi finora. 'Papà va in TV' apre la raccolta L'ultima lacrima (1994), una collezione eclettica di ventisette pezzi, molto vari per lunghezza, stile e riuscita. La raccolta è stata definita da Lunetta (1995:111) "un'arma che non appena ha esploso un proiettile-racconto, viene ricaricata al racconto successivo, in una giostra pirotecnica di trovate al vetriolo e di soluzioni brutalmente inattese." Qui l'autore sembra lasciare libere le redini della sua fervida immaginazione narrativa senza un preciso principio organizzativo, che non sia appunto il sarcasmo, o "l'umor nero di Benni" come lo ha definito Ajello (1994:31). Questo cattivo umore, quasi una patina nera che avvolge tutti i racconti, è significativamente trasfigurato in umorismo nero nel primo racconto, che simbolicamente stabilisce il tono dell'intera raccolta.

Il racconto 'Papà va in $\mathrm{TV}^{, 15}$ ritrae la famiglia Minardi che si riunisce con i vicini in occasione dell'apparizione del signor Minardi alla televisivione. Il pezzo si apre con i premurosi preparativi della signora Lea, mostrando poi l'eccitazione e l'orgoglio di tutti per essere tra quelli che possono 'vantare' di avere un amico o familiare apparso in TV. Come in un montaggio cinematografico, anche il signor Minardi, preoccupato di far bella figura, viene 'ripreso' mentre si rammarica di non aver potuto lavare i denti. L'estrazione popolare di ambientazione e personaggi distrae inizialmente l'attenzione del lettore, ma la satira benniana non vuole in realtà prendere di mira la dabbenaggine della gente

15 L'ultima lacrima, Milano: Feltrinelli, 1994: 9-17. D'ora in poi Lacrima. 
bensì il potere disumanizzante della società mediatica. La struttura reticente e la sottile ambiguità del dialogo nascondono, nella prima parte del pezzo, il vero motivo del fermento e dell'emozione, ovvero l'esecuzione del signor Minardi in diretta. Questo ermetismo contribuisce a creare una sinistra atmosfera di attesa:

[Mario, il vicino] saluta i bambini e stringe con energia la mano a Patrizio.

Allora, campione, contento del tuo papà?

Insomma...- fa Patrizio.

Che bella pettinatura - dice Mariella a Lea - ci siamo fatte belle eh, oggi? Già, non è un giorno come tutti gli altri.

In un certo senso...- fa Lea. (Lacrima: 11)

L'ambiguità prosegue all'apparizione del signor Minardi sul teleschermo, che strappa ai familiari e amici a casa "un grande applauso e anche qualche lacrima." Anche in questa occasione la polisemia dell'espressione non è disambiguata, mantenendo il doppio senso di espressione di gioia o di dolore.

La seconda parte del racconto si concentra sull'evento televisivo, la prima esecuzione in diretta nella storia del paese, e sull'eccitazione del mondo mediatico. I toni enfatici del presentatore, le chiacchiere e gli insulti degli invitati, le interruzioni pubblicitarie che seguono sono una spietata satira della TVspazzatura degli ultimi anni. La chiusura del racconto, che vede la giovane figlia al telefono rispondere alle domande del paparazzo di turno, fornisce la degna conclusione allo sconcertante racconto: "Mio fratello sta facendo dei salti sul divano, il signor Mario sta bevendo il whisky, la mamma piange con la testa sulle ginocchia 
della signora Mariella. Molto? Sì, mi sembra che pianga molto. Io? Io sto al telefono con lei, no? Sì, mi chiamo Lucilla, mi raccomando con due elle, non Lucia, che a scuola si sbagliano sempre..." (Lacrima: 17).

Il bersaglio della satira benniana, la televisione e i sui effetti inebetenti, è uno di quelli che ricorrono con maggiore frequenza nei suoi lavori. A partire dai pezzi su il manifesto Benni ha attaccato il populismo e la dabbenaggine della TV italiana e ne ha deriso il suo pubblico, la cui divertente tassonomia è presentata nel corsivo di quegli anni 'Applaudi, cretino', che si chiude ad effetto con un'amara conclusione:

Invano abbiamo atteso da lui [pubblico da studio], in tanti anni, un piccolo segno di ribellione. Non dico un fischio o un pomodoro, ma almeno un dignitoso silenzio a una barzelletta preistorica, uno sgambetto a Don Lurio lanciato tra le file [...] Neanche li abbiamo mai sorpresi, una volta, col dito nel naso. Ci dispiace. Perché questo pubblico di marziani, che la TV fa applaudire, e ridere, e alzarsi e risedere a comando, assomiglia tanto a tutti noi. $^{16}$

Naturalmente Benni ha ironizzato molto sulla censura e strumentalizzazione della TV fattane a suo tempo dal partito di maggioranza, la Democrazia Cristiana, definita "il partito col più alto indice di assolto" ${ }^{\prime 7}$ e l'occasione è quasi sempre offerta dall'attualità o da qualche evento televisivo. ${ }^{18}$

16 'Applaudi, cretino', Aprile 1978, in Spettacoloso, Milano: Mondadori, 1981: 15.

17 'Mike Gary Bongiorno III', Marzo 1979, ibidem: 43. 
La profonda avversione nei confronti della TV nazionale si acutizza negli anni seguenti e, oltre che negli scritti, si realizza anche in una programmatica assenza dal video. È soprattutto nei romanzi che Benni trova lo spazio narrativo per approfondire con la consueta fantasia il tema del potere manipolativo dei mass-media. Nel distopico mondo di Baol (1990) si parla di "realtà composta", ovvero il risultato della mescolanza di fiction e "realtà primaria" di cui sono fatte le trasmissioni del regime. Nell'orwelliano Elianto (1996) il potere pervasivo dei media è ormai una dittatura vera e propria: ogni singola attività nel paese di Tristalia è governata da un computer centrale che garantisce il consenso dei cittadini attraverso programmi a quiz giornalieri, somministrando punizioni, ovvero niente erogazione di luce, acqua ecc., a quelle famiglie che non si dimostrano informate sulle idee del governo (e dunque danno risposte sbagliate). Un'idea simile era già stata elaborata narrativamente da Benni nel racconto 'Un cattivo scolaro' (L'ultima lacrima, 1994). Qui, in una sorta di scuola dell'obbligo televisivo, lo studio della storia della televisione ha sostituito quello della letteratura e chi non segue il telegiornale di propaganda viene mandato in istituti di rieducazione. La scuola è finanziata con gli slogan pubblicitari, che passano agli altoparlanti, e gli studenti diligenti possono scegliere tra un buon voto o il gadget dello sponsor.

Come ho suggerito in precedenza, la forma del racconto e del corsivo appare a mio avviso più congeniale all'arte di Benni per la maggiore disciplina che essa impone all'esuberanza, a volte incontrollata, della sua scrittura. Il racconto breve è in ogni caso 
utilizzato da Benni per sviluppare un'intuizione o un tema da rielaborare per i romanzi, anche se fra quelli pubblicati, la gran maggioranza presentano caratteristiche strutturali o di eleborazione linguistica che ne fanno pezzi a se stanti.

Quello che mi sembra interessante sottolineare al proposito invece è la tendenza di Benni a giocare con i generi letterari, quasi a voler 'testare' una stessa idea attraverso forme diverse, o viceversa piegare i vari generi alle sue esigenze di critica sociale e politica. L'avversione alla televisione è infatti espressa anche in versi nel componimento 'Estate alternativa' ${ }^{19}$ e il genere della favola, di cui ho parlato prima, è duplicemente stravolto: in Terra! , fra i vari racconti nel racconto, troviamo una rivisitazione della favola di Cappuccetto Rosso, in cui la protagonista, Cappuccetto Nero, è una giovane trafficante del Bronx. Una versione simile compare, sotto forma di rap, nella raccolta Ballate (1991). Questa volta l'ambientazione è la Bologna della mobilitazione e della protesta del '77:

\section{'Little Red Hood'}

ovvero: vera storia di Cappuccetto Rosso per bambini metropolitani da leggere a tempo di musica schioccando le dita

La favola ragazzi che vi voglio raccontare è la storia di una pupa che sapeva cosa fare

19 'Rimpiango Tognazzi e la sua bella faccia/a sua modestia e la sua stanchezza/vorrei che morissi della tua battutaccia/comicuzzo cortigiano, euforica schifezza/Fessi telegenici e fascistelle annoiate/assessori col grano, piazze illuminate/quattro cazzate, una piazza d'estate/un buffet leggero, comici e insalate'. In Ballate, Milano: Feltrinelli, 1991 (1993): 111. 
in giro la notte da sola nel bosco

il suo nome era Cappuccetto Rosso

era una sbarbina col capello corto bionda

lavorava sostituta commessa alla Standa

abitava in una vecchia casa occupata

col soffitto di marzapane e le finestre di cioccolata

con Hansel e Gretel due punk meridionali

e un gatto sadomaso con gli stivali $[\ldots]^{20}$

In perfetto stile riciclatorio post-moderno, Benni ritorna alle fonti multimediali della sua ispirazione e ne rielabora forme e contenuti in un gioco intertestuale continuo e in 'esercizi di stile' da cui i suoi stessi testi sono tuttaltro che esclusi. In questa fagocitazione onnivora e in questo gioco letterario i confini fra racconto o ballata, corsivo o monologo teatrale sono ormai del tutto sbiaditi.

20 In Ballate, op. cit.: 119. 


\section{BIBLIOGRAFIA}

\section{Fonti primarie: Opere di Stefano Benni}

Romanzi

1983 Terra! Milano: Feltrinelli.

1986 Comici spaventati guerrieri. Milano: Feltrinelli.

1990 Baol. Una tranquilla notte di regime. Milano: Feltrinelli.

1992 La compagnia dei Celestini. Milano: Feltrinelli.

1996 Elianto. Milano: Feltrinelli.

2000 Spiriti. Milano: Feltrinelli.

2001 Saltatempo. Milano: Feltrinelli.

2003 Achille Pié Veloce. Milano: Feltrinelli.

2005 Margherita Dolcevita. Milano: Feltrinelli.

2009 Pane e tempesta. Milano: Feltrinelli.

Corsivi

1977 La tribù di Moroseduto. Milano: Mondadori.

1979 Il Benni furioso. Roma: il manifesto.

1979 Non siamo stato noi. Roma: Savelli. 
1981 Spettacoloso. Milano: Mondadori.

1986 Il ritorno del Benni furioso. Roma: Coop. il manifesto ' 80 .

2001 Dottor Niù. Corsivi diabolici per tragedie evitabili. Milano: Feltrinelli.

\section{Racconti}

1976 Bar Sport. Milano: Mondadori.

1984 I meravigliosi animali di Stranalandia. Milano: Feltrinelli. (illustrazioni di Pirro Cuniberti).

1987 Il bar sotto il mare. Milano: Feltrinelli.

1994 L'ultima lacrima. Milano: Feltrinelli.

1997 Bar Sport Duemila. Milano: Feltrinelli.

2007 La grammatica di Dio. Milano: Feltrinelli.

Poesia e Teatro

1981 Prima o poi l'amore arriva. Milano: Feltrinelli.

1991 Ballate. Milano: Feltrinelli.

1998 Blues in sedici. Ballata della città dolente. Milano: Feltrinelli.

1999 Teatro . Milano: Feltrinelli. 
2003 Teatro II. Milano: Feltrinelli.

\title{
Fonti secondarie
}

\author{
Ajello, N. 1994 Benni al veleno. La Repubblica, 20 ottobre:31. \\ Benni, S. \& Fofi, G. 1999 Leggere scrivere disobbedire. Conversazione con \\ Goffredo Fofi. Roma: minimum fax. \\ Bertoni, R. \\ 2000 Aspetti del fantastico in alcuni romanzi italiani \\ degli anni '80-'90. Italia \& Italy, 7:3-7. \\ Capozzi, R. \\ 1993 Apocalittici e integrati nell'industria culturale \\ postmoderna. In: Capozzi, R., Ciavolella, M. \\ (eds.) Scrittori tendenze letterarie e conflitto delle \\ poetiche in Italia (1960-1990). Ravenna: Longo: \\ 205-222.
}

Ceserani, R.

1994 Modernity and postmodernity: a cultural change seen from the Italian perspective. Italica, 71:369384.

Clark, T.

2004 Not Seeing the Short Story. A Blind Phenomenology of Reading. The Oxford Literary Review, 26:5-30.

Degli Esposti, C. 1995 Interview with Stefano Benni: a post-modern moraliste. Italian Quarterly, 32: 99-105. 
De Meijer, P.

Goldberg, M.E.

Guagnini, E.

Guglielmi, G.

Gullason, T.A.

Hallett, C.J.

Jacobs, H.C.

La Porta, F.

Lunetta, M.
1984 La prosa narrativa moderna. In: Asor Rosa, A. (ed.) Letteratura italiana. Le forme del testo. La prosa. Torino: Einaudi: 759-847.

1996 The Synchronic Series as the Origin of the Modernist Short Story. Studies in Short Fiction, 33:515-527.

1993 Il racconto breve italiano del Novecento. In: Alonso Hernández, J.L., Gosman, M., Rinaldi, R. (eds.) La Nouvelle Romane. Italia France Espańa. Amsterdam/Atlanta: Rodopi: 115-131.

1989 Esiti novecenteschi della novella italiana. In: $L a$ Novella Italiana. Atti del Convegno di Caprarola 19-24 Settembre 1988. Roma: Salerno Editrice: 607-625.

1989 What Makes a 'Great' Short Story Great?. Studies in Short Fiction, 26:267-277.

1996 Minimalism and the Short Story. Studies in Short Fiction, 33:487-495.

1997 Original und Pastiche in Wort und Bild. Italienisch, 38:21-37.

1995 La nuova narrativa italiana. Travestimenti e stili di fine secolo. Torino: Bollati Boringhieri.

1995 Nunc scribendum est' diceva Agostino di Ippona. Il Ponte, 51:104-114. 
Lyall, R.J.

Magistà, A.

Malatesta, S.

May, C.E.

May, C.E. (ed.)

May, C.E.

May, C.E.

Scarsella, A.

Schiavo, C
1999 Intimations of Orality: Scotland, America and the Early Development of the Short Story in English. Studies in Short Fiction, 36:311-325.

1997 Il vendicatore invisibile. Il Venerdì di Repubblica, 29 agosto:67.

1992 Cari comici siete a pezzi. La Repubblica, 14 ottobre:27.

1984 The Nature of Knowledge in Short Fiction. Studies in Short Fiction, 21:327-338.

1994 The New Short Story Theory, Athens, Ohio: Ohio University Press.

1995 The Short Story. The Reality of Artifice, New York: Twayne.

1996 Prolegomenon to a Generic Study of the Short Story. Studies in Short Fiction, 33:461-473.

1995 Il racconto fantastico italiano nel secondo dopoguerra. Punti di vista per una definizione trasversale. In: Van Volsen, S., Musarra, F., Van den Bossche, B. (eds), I tempi del rinnovamento. Atti del Convegno Internazionale 'Rinnovamento del codice narrativo in Italia 1945-1992'. Roma: Bulzoni \& Leuven University Press: 373-385.

2002 Il racconto italiano di fantascienza negli anni Novanta. Bollettino '900, I-II, www.unibo.it/boll900/numeri/2002-i/Wbol/Schiavo/Schiavo_frame.html. 
Segre, C.

Siciliano, E.

Sinibaldi, M.

Tani, S.

Turchetta, G.

Trussler, M.
1989 La novella e i generi letterari. In: La Novella Italiana. Atti del Convegno di Caprarola 19-24 Settembre 1988. Roma: Salerno Editrice: 47-57.

1983 Premessa. In: Racconti italiani del Novecento. Milano: Mondadori: 11-28.

1997 Pulp. La letteratura nell'era della simultaneità. Roma: Donzelli.

1990 Il romanzo di ritorno. Dal romanzo medio degli anni sessanta alla giovane narrativa degli anni ottanta. Milano: Mursia.

2004 L'ambigua 'serenità del brutto'. Appunti per una tipologia del racconto comico. In: Merola, N., Rosa, G. (eds.) Tipologia della narrazione breve. Atti del Convegno di Studio 'Il Vittoriale degli Italiani'. Gardone Riviera 5-7 giugno 2003. Manziana (Roma): Vecchiarelli: 101-136.

1996 Suspended Narratives: The Short Story and Temporality. Studies in Short Fiction, 33:557577. 